\title{
ACTyourCHANGE Study Protocol. The Promotion of Healthy Lifestyle for Obese Patients with Acceptance and Commitment Therapy provided with A Modular Approach.
}

Anna Guerrini Usubini ( $\square$ anna.guerriniusubini@unicatt.it )

Unicatt

Roberto Cattivelli

Istituto Auxologico Italiano Istituto di Ricovero e Cura a Carattere Scientifico

Emanuele Giusti

Istituto Auxologico Italiano Istituto di Ricovero e Cura a Carattere Scientifico

Francesco Vailati Riboni

Universita Cattolica del Sacro Cuore

Giorgia Varallo

Istituto Auxologico Italiano Istituto di Ricovero e Cura a Carattere Scientifico

Giada Pietrabissa

Istituto Auxologico Italiano Istituto di Ricovero e Cura a Carattere Scientifico

Gian Mauro Manzoni

Istituto Auxologico Italiano Istituto di Ricovero e Cura a Carattere Scientifico

Simone Consoli

Istituto Auxologico Italiano Istituto di Ricovero e Cura a Carattere Scientifico

Ilaria Bastoni

Istituto Auxologico Italiano Istituto di Ricovero e Cura a Carattere Scientifico

Valentina Granese

Istituto Auxologico Italiano Istituto di Ricovero e Cura a Carattere Scientifico

Clarissa Volpi

Istituto Auxologico Italiano Istituto di Ricovero e Cura a Carattere Scientifico

Valentina Villa

Istituto Auxologico Italiano Istituto di Ricovero e Cura a Carattere Scientifico

Annalisa Caretti

Istituto Auxologico Italiano Istituto di Ricovero e Cura a Carattere Scientifico

Michela Bottacchi

Istituto Auxologico Italiano Istituto di Ricovero e Cura a Carattere Scientifico

Gianluca Castelnuovo

Istituto Auxologico Italiano Istituto di Ricovero e Cura a Carattere Scientifico 


\section{Enrico Molinari}

Istituto Auxologico Italiano Istituto di Ricovero e Cura a Carattere Scientifico

\section{Study protocol}

Keywords: Acceptance and Commitment Therapy (ACT), Focused-Acceptance and Commitment Therapy (FACT) Psychological flexibility, Cognitive Behavioral Therapy (CBT), Healthy Lifestyle, Obesity

Posted Date: February 2nd, 2021

DOI: https://doi.org/10.21203/rs.3.rs-47403/v1

License: (c) (1) This work is licensed under a Creative Commons Attribution 4.0 International License. Read Full License

Version of Record: A version of this preprint was published at Trials on April 20th, 2021. See the published version at https://doi.org/10.1186/s13063-021-05191-y. 


\section{Abstract}

Background: Acceptance and Commitment Therapy (ACT) resulted to be efficacious in promoting the core process of Psychological Flexibility, a key ability related to physical and psychological health outcomes. Despite evidence-based ACT protocols were applied successfully in different contexts, including the promotion of long-standing behavioral change, the impact of the single processes in the psychological flexibility model remains unclear. The aim of the present study is to evaluate the efficacy of a Focused-ACT intervention for the maintenance of a healthy lifestyle, by separating and evaluating the impact of single core processes targeted into a modular intervention on the maintenance of healthy lifestyle.

Methods: An individually randomized group treatment trial will be conducted. 150 adult obese patients who are attending a four-week medically-based multidisciplinary rehabilitation of weight loss will be randomly allocated into three groups: Module Engage, Module Openness, and Module Awareness. At the beginning (Time 0 ) and at the end of the intervention (Time 1), at six months (Time2) and one year (Time 3) follow-up weight and height will be recorder and participants will complete the Psychological General Well Being Inventory(PGWBI), the Outcome Questionnaire-45.2 (OQ-45.2), the Brief Values Inventory (BVI), the Committed Action Questionnaire (CAQ), the Italian-Cognitive Fusion Questionnaire (I-CFQ), the Acceptance and Action Questionnaire (AAQ II) and the Five Facet Mindfulness Questionnaire (FFMQ).

Repeated measures 3 (conditions) x 4 (times) will be assessed to examine differences between three groups within four times on both general outcomes measure of weight, BMI, PGWBI and OQ-45.2, and FACT processes targeted during the interventions.

Discussion: By providing additional evidence supporting the relevance of modular transdiagnostic interventions in clinical practice and the use of Process-Based Therapy, this study will contribute to clarify which mechanisms are involved in a generalizable lifestyle behavioral change intervention.

\section{Trial registration}

ClinicalTrials.gov Registration number: NCT04474509 Date: July, 42020 https://clinicaltrials.gov/ct2/show/NCT04474509

\section{Background}

Acceptance and Commitment Therapy (ACT; Hayes, Luoma, Bond, Masuda, \& Lillis, 2006) is one of the recent third-wave Cognitive Behavioral Therapy (CBT) raised in the last twenty years. It is grounded in Functional Contextualism (Biglan \& Hayes, 1996; Hayes \& Wilson, 1994) a form of pragmatism based on the principle of "act in the context", that emphasizes the role of context in understanding the nature and the functions of events that occur. Based on behavioral therapy, ACT is also theoretically rooted in Relational Frame Theory (RFT; Hayes, Gifford, \& Ruckstuhl Jr, 1996), a contextual theory of cognition that 
conceptualizes human psychological suffering as resulting from the relationship between individuals and their historically and situational-based context.

ACT targets six core processes to promote "psychological flexibility" defined as "contacting the present moment fully as a conscious human being, and basing on what the situation affords, changing or persisting in behavior in the service of chosen values" (Hayes et al., 2006): Acceptance, Defusion, Contact with the present moment, Self as a context, Values, Committed Actions. These processes were condensed in the Focused- Acceptance and Commitment Therapy approach (FACT; (Strosahl, K, Robinson, \& Gustavsson, 2012) a brief application of ACT-based intervention, which is characterized by three core components:

- Openness refers to the willingness to experiencing distress and undesirable private events as part of human experience, without judgment or attempts to avoid or control internal states, even if they are unpleasant. It encompasses the promotion of cognitive defusion, that concerns the relationship between a person and his internal states, including thoughts, feelings, and physical sensations. While a "fused" person acts as thoughts are literally true, instead of perceiving them as mental events (Hayes, Levin, Plumb-Vilardaga, Villatte, \& Pistorello, 2013), defusion allows distancing from own personal internal states and watch them as objects.

- Awareness is the ability to be present at the moment and face events as contextually situated. To be aware means stepping back from suffering situations and seeing them in the context where they occur. This kind of acceptance is a valid alternative to Experiential avoidance, that is one of the most important pathological processes that allows people to avoid unpleasant internal events, thoughts, feelings, physical sensations, even though the attempt to escape causes psychological distress.

- Engagement in actions linked to personal values, such as relationships, work, spiritual growth. If a person engages himself in committed actions driven by chosen life directions is able to pursue a meaningful and coherent life.

FACT resulted in a promising evidence-based behavioral intervention, particularly suitable to health care settings, due to his brief nature and his adaptation in group settings (Glover et al., 2016).

Glover and colleagues, (2016) provided findings in support of the efficacy of a brief - 4 group sessions FACT protocol in producing improvement of perceived quality of life and reducing stress, anxiety, and depressive symptoms in 51 patients in a primary care setting.

Since Psychological Flexibility model is complex, it could be interesting to study the specific role of every single sub-component abovementioned, in order to deepen the effect of Psychological Flexibility on health and well-being (Levin, Herbert, \& Forman, 2017; Levin, Krafft, \& Twohig, 2020).

To do so, one possible approach is the modularization of treatment provided to foster Psychological Flexibility into specific modules each of whom related to every single sub-component. 
Modularization has been described as an attempt to break complex activities in single components that act independently. A single module is a unit within a whole intervention that is expected to produce an intended result in therapy, while interacting with each other component of the structure, but maintaining his function limited to the module, without interfering with others.

Unpacking the manualized intervention into specific modules allows therapists to personalize intervention for the need of individuals, while preserving the standardization of therapy, according to evidence-based guidelines (Chorpita et al., 2013; Villatte et al.,2016).

With this work, we propose a treatment protocol based on the application of a FACT- oriented group psychological treatment, applied in a single health care center for obesity management. The main scope of the intervention is to promote some crucial psychological abilities for stable behavioral change, to foster the long-term adoption of a healthy lifestyle, in obese patients undergoing treatment for weight management, according to the FACT model.

The aim of the present study is twofold: The first aim is to evaluate whether our intervention is effective in producing noticeable changes in processes targeted within the modules. The second clinically-relevant interest is to test the specific impact of each single core process of FACT on the ability to change behaviors permanently, in order to maintain a healthy lifestyle.

Due to the novelty of our intention, it is difficult to perform well-defined hypotheses. The only expectation is to find the pre-to-post intervention improvement in psychological processes, given by the effect of each module provided, and a general change in treatment outcomes.

\section{Methods}

\section{Study design}

A three-arm Individually Randomized Group Treatment Trial (IRGT)will be conducted (Andridge, Shoben, Muller, \& Murray, 2014; Pals et al., 2008). Participant enrolled for the study will be randomly allocated into three experimental conditions attended during the inpatient phase:

1. Module Engage

2. Module Openness

3. Module Awareness.

At the beginning (Time 0), at the end of intervention (Time 1), after 6 months (Time 2) and 12 months (Time 3) follow-up, weight, height and the battery of questionnaires addressing outcome and process measures will be collected.

Also, at discharge, they will receive wearable devices for the daily recording of habits regarding diet and physical activity. They are asked to wear devices for one month just after discharge in order to test the ability to follow over time the medical recommendations and maintain long-standing healthy habits. 
During this period, all the participants are monitored, with the support of technology, using wearable devices and mobile app to measure their healthy habits.

\section{Participants}

Participants will be obese patients enrolled for a weight loss rehabilitation program in a single clinical center in the North of Italy. Patients will be included if they will meet these following inclusion criteria: 1) age between 18 and 65 years; 2) BMI>30;3) clinical recommendation for the group psychological treatment (basing on the internal procedures of the structure described in the Procedures section) 4) written and informed consent to participate; 5) being technology friendly to use wearable devices.

Exclusion criteria for the study are: 1) other psychiatric disturbances (diagnosed according to DSM 5 criteria); 2) other medical conditions not related to obesity that could compromise participation at the study.

\section{Measure}

\section{Outcome measures}

The primary outcome of the study is weight loss maintenance. Weight, and height will be assessed to calculate Body Mass Index $\left(\mathrm{BMl}=\mathrm{kg} / \mathrm{m}^{2}\right)$. Success in weight loss is achieved if individuals lose at least $5 \%$ of initial weight at the end of treatment and maintains the percentage of weight lost for at least one year (Wing \& Hill, 2001).

The second outcome measure is psychological well-being. The Psychological General Well-Being Inventory (PGWBI; (Dupuy, 1984) Italian validation of Grossi and colleagues (Grossi et al., 2006) consists of 22 self-administered items rated on a 6-point Likert scale, relative to six subscales that offer a measure of the level of subjective psychological well-being. Subscales are anxiety, depression, positive well-being, self-control, general health, and vitality with a range of Alpha's scores from 0.61 to 0.85 for each subscale.

The third outcome measure is the outcome of psychological treatment. The Outcome Questionnaire-45.2 (Lambert, Gregersen, \& Burlingame, 2004) Italian version by Chiappelli, Coco, Gullo, Bensi, e Prestano (2008) as a measure for the assessment of psychological treatment. It is a self-report questionnaire composed of 45 items. Subscales are symptoms distress, interpersonal relations, and social role functioning. The total Alpha score is excellent (.90 for clinical sample; .92 for non-clinical sample).

\section{ACT Processes measures}

\section{Module Engagement}

The Brief Values Inventory (McCracken \& Yang, 2006) Italian validation of Baroni, McCracken, Matera, Nerini, \& Stefanile (2019). It is composed by 12 items aimed to assess the success in living according to 
personal values. The internal consistency of the Success subscale is .70 in the Italian validation study.

The Committed Action Questionnaire (McCracken, 2013) Italian adaptation of Baroni, Matera, Nerini, \& Stefanile ( 2017) is 8-items self-report questionnaire rated on 7-point Likert scale, used to assess positive and negative aspects of the ability to engage themselves into committed actions driven by values. The internal consistency of the measure tested on a normative sample was good $(a=.80)$.

\section{Module Openness}

The Italian-Cognitive Fusion Questionnaire (Gillanders et al., 2014) Italian version by Oppo and colleagues (2019) is 7-items questionnaire administered for the assessment of cognitive fusion. The internal consistency of the Italian version is excellent $(a=.82)$.

The subscale "Nonjudge" of the Five Facet Mindfulness Questionnaire, described below, is used as a measure of Acceptance. The internal consistency of the subscale is . 86

\section{Module Awareness}

The Five Facet Mindfulness Questionnaire (FFMQ; Baer, Smith, Hopkins, Krietemeyer, \& Toney, 2006). The Italian version (Boffito et al., 2009) is 39-item-self-report questionnaire used as a measure of Mindfulness. It is composed by five subscales: observe, describe, act with awareness, non-react, nonjudge. The internal consistency of total scale is good (a .86).

Psychological flexibility

The Acceptance and Action Questionnaire (AAQ II; Bond et al., 2011). The Italian version (Pennato, Berrocal, Bernini, \& Rivas, 2013) is the most widely used self-reported questionnaire that measures psychological inflexibility and experiential avoidance. It is composed by 10 items, rated on 7-point Likert scale, the Internal consistency is good ( $a .83$ ) and test-retest reliability is moderate (.61)

\section{Monitoring measure}

Another measure concerns healthy lifestyle monitoring relative to the period of one month after discharge: Indicators are: 1) total amount of moderate-to-vigorous physical activity in minutes; 2) weekly mean of daily total amount of calories; 3 ) weekly mean of total daily steps.

All data collected will be stored in cloud computing and provided to researchers through a dedicated web platform.

\section{Procedures}

At the beginning of the study (Time 0), weight and height of participants will be assessed to calculate Body Mass Index (BMI: $\mathrm{Kg} / \mathrm{m}^{2}$ ) and PGWBI, OQ-45.2 BVI, CAQ, I-CFQ, AAQ II and FFMQ will be administered. 
Accordingly to recent guidelines for the management of adult obesity (Giusti et al., 2020; Yumuk et al., 2015), all patients will attend the rehabilitation program composed by medical, nutritional physical and psychological components. Patients will follow a hypocaloric balanced diet provided by dietitians (energy intake around $80 \%$ of the basal energy expenditure estimated, according to the Harris-Benedict equation). They will take part in a nutritional counseling program provided both in individual and group sessions focused on nutritional education, information on obesity and their health-related risks, setting personal goals for weight loss, weight management, behavioral changes, and prevention of relapses.

The patients will perform physical activity once a day (one hour) with trainers and physiotherapists consisting of programs of aerobic activity, postural gymnastics, and walking. They also will receive psychological assessment provided by clinical psychologists expert in health psychology, aimed to assess the individual characteristics including physical and psychological well-being of the patients, the possible presence of Eating Disorders on any other psychological disturbances including mood dysregulation, anxiety, depression, investigate the presence of familial and social support, the level of motivation of change and the willingness to engage themselves into a stable lifestyle habits change. Basing on their needs, patients will receive psychological support provided both in individual and in group settings.

Patients enrolled for the study will follow followed the medical, nutritional and physical rehabilitation program, while with respect to the psychological component, they will be randomly allocated into three experimental conditions:

\section{Module Engage \\ 2. Module Openness \\ 3. Module Awareness.}

A detailed description of the condition will be provided as follow.

The randomization will be performed using the proper web site (www.randomization.com).

All conditions comprise 2 group sessions, provided twice a week, lasting about one hour each. Sessions will be delivered by an expert clinical psychologist in ACT and will include experiential exercises and metaphors. A detailed description of the sessions will be included in the following section.

At the end of the treatment (Time 1), after 6 (Time 2) and 12 months (Time 3 ) the outcome and process measures (PGWBI, weight, BMI, OQ-45.2, BVI, CAQ I-CFQ, AAQ-II and FFMQ will be repeated. During the inpatient phase the measures will be directly administered, while at 2 points follow-up data will be collected via online survey.

Participants also received wearable devices and will be instructed on how to use wearable for the following one month, after discharge. 
Adverse events or other unintended effects of trial intervention could be managed by the researchers involved in the study.

Those who suffer from trial participation can consult the psychologist responsible for the hospital division.

Completed informed and written consent will be delivered to the responsable of the study.

All personal information about participant and records of all patients will be collected and preserved separately, in a secure place, into password-protected files kept for five years after the end of trial

The Medical Ethic Committee of Istituto Auxologico Italiano approved the study.

\section{Intervention}

\section{ACT Module on Engagement}

During this module, patients will have the opportunity to increase their motivation to change and encourage the engagement in committed actions, consistent with their life values. Patients are invited to reflect on what is important in their lives, which values make their life worth living, and which actions they could take to live a meaningful life, in accordance with personal values. The use of metaphors and experiential exercises will facilitate the process of exploring personal values, identifying life directions and related behaviors. For example, the $80^{\text {th }}$ Birthday Party metaphor requires participants to imagine there is a party in honor of their birthday and the time comes when people are starting to give speeches and try to answer the question about what they want to hear people at the party say. This exercise help patients in wondering what person they want to be with themselves and others.

\section{ACT Module on Openness}

Participants attending this module are guided to recognize and distancing themselves from stressful thoughts, feelings and sensations. They will learn to read suffering as part of human experience, without self-judgment and self-condemnation. Rather, the psychologist will encourage the patient's assumption of an open and acceptable approach to internal experiences. Throughout the module, the psychologist will help patients to reflect on their usual, but ineffective efforts to solve personal problems, and encourage the adoption of new responsive strategies based on acceptance and defusion from personal distress.

An example of a metaphor used during the Module is The Passenger on a bus. In this metaphor, patient have to imagine to be a driver bus and his every thought is a passenger that gets on and off the bus. This exercise help patients to accept, defuse from, and reduce the power of their thoughts.

\section{ACT Module on Awareness}

The module comprises meditation exercises and experiences aimed to learn how to act intentionally with awareness about personal thought and sensations without automatically reacting. Participants are 
supported to recognize their actions and the context where they occur and learn to choose to respond with action consistent with their values and not automatically. The psychologist

will propose breathing exercises, body scan and others mindfulness experiences. Participants will be encouraged to sitting comfortably, close the eyes, feel themselves in contact with the present moment they are living, paying attention to their breath, noticing the rhythm and any other aspect of the experience of breathing. Then, the therapist guides the participant's attention on the body, noting any part of their body from the head to feet. Then, the sounds around, any noises that could distract their attention on themselves.

\section{Treatment fidelity}

The research group comprises licensed psychologists, researchers, and doctoral student experts in the field of clinical interventions in health care settings and research. Group sessions will be administered by a clinical psychologist with many years of expertise in ACT clinical practice both in individual and group settings, blinded to research scopes. The structure and the content of modules are consistent with ACT theory. Two bachelors-level observers, also blinded to research, after a period of training, will attend at least $20 \%$ of sessions to evaluate the adherence to the protocol, the coverage of contents and the use of any additional strategies. They will use a checklist detailed for all the content of each module, including the process targeted, experiential exercises and metaphors planned for each session. Coders have to achieve a minimum of $80 \%$ reliability with the expert trainers and each other. With lower level of agreement, the data will be dismissed. (Villatte et al., 2016).

\section{Statistical analysis}

Sample size has been calculated for a 3 (between) x 4 (within) ANOVA using G*Power software (release 3.1.3) (Faul, Erdfelder, Lang, \& Buchner, 2007). Setting alpha to 0.05 , power to 0.80 , the correlation among repeated measures to 0.5 and the non-sphericity correction to 1 , a total sample of 45 participants are required to detect a small-size interaction effect $(f=0.20)$.

Taking into account a possible correlation among observation within groups measured in a previous study (Interclass Correlation Coefficient: ICC $=0.15$; Cattivelli et al., 2020), a number of participant per groups of 7, the variance inflation factor (Donner, Birkett, \& Buck, 1981) was 1.9

With a possible dropout of $10 \%$ at follow-up, the final number of participants needed for the study is 90 .

$[(45 \times 1.9)+10 \%]$

Data will be analyzed on an Intention-To-Treat basis using a 3 (conditions) x 4 (times) repeated measure Analysis of Variance (ANOVA) to examine differences between three groups within four times on both general outcomes measure of weight, BMI, PGWBI and OQ-45.2, and ACT processes targeted during the interventions (BVI, CAQ, I-CFQ, and FFMQ) including the general measure of Psychological Flexibility 
(AAQ II). Corrected effect sizes (Hedges's g) and significance at 95\% confidence interval (CI 95\%) will be calculated for both between-group and within group differences.

Analysis will be performed using IBM Statistical Package for the Social Sciences (SPSS) version 26.

\section{Discussion}

The current study aims to provide additional evidence supporting the relevance of promoting Psychological Flexibility in order to foster healthy lifestyle and improve general subjective well-being through a modular transdiagnostic intervention suitable for clinical practice.

Modularization makes the intervention more practical, integrative, flexible, and patient-centered. Also, the modular treatment allows clinicians to suit the intervention with their expertise and the need of patients (Rotheram-Borus, 2012) by orienting therapist in selecting the modules (Chorpita, Daleiden, \& Weisz, 2005).

Modularized treatment is also coherent with the raising interest for the Process-based research focused on understanding the processes involved in human suffering rather than addressing symptoms reduction.

The present study is quite innovative for different reasons. Firstly, to the author's knowledge, this is one of the first attempts to evaluate the impact of single targeted processes of FACT on affecting healthy lifestyle change. Focusing on the mechanism of change could clarify which factors are involved in a generalizable lifestyle behavioral change intervention.

Another relevant strength is determined by the experimental design of the study. The IRGT for the evaluation of the effectiveness of interventions is characterized by the randomization of individuals into experimental conditions in which treatment is delivered in groups. These designs are very common in the field of public health because they are practice and less expensive and take into account the influence of the group environment where the intervention is provided (Pals et al., 2008).

It is important to notice also the advantages of using wearable devices in the outpatient phase, as a direct measure of healthy habits followed by patients in their daily life context.

Nonetheless, several limitations must be pointed out. This study will not include the control group or comparison with another intervention.

In addition, IRGT requires proper analytic methods that should take into account the influence of a potential correlation among observations within treatment conditions (Pals et al., 2008).

Due to the relatively small number of sessions and previous evidence suggesting the non-significance effect of the treatment groups (Cattivelli et al., 2020) found in a similar previous study, we assume to adopt a traditional statistical analysis method, even if the assumption of the independence of the 
observation is violated. To deal with this limitation, the variance inflation factor was taken under consideration in sample size calculation.

Another limitation is represented by the highly specific context where the study will take place, limiting the external validity of the research.

Despite these limitations, the study could represent an important advancement in the field of the theoretical development of the Psychological Flexibility model, with also relevance for the clinical practice.

\section{Abbreviations}

\begin{tabular}{ll} 
CBT & Cognitive Behavioral Therapy \\
\hline ACT & Acceptance and Commitment Therapy \\
\hline FACT & Focused- Acceptance and Commitment Therapy \\
\hline IRGT & Individually Randomized Group Treatment Trial \\
\hline BMI & Body Mass Index \\
\hline PGWBI & Psychological General Well Being Inventory \\
\hline OQ-45.2 & Outcome Questionnaire-45.2 \\
\hline BVI & Brief Values Inventory \\
\hline CAQ & Committed Action Questionnaire \\
\hline I-CFQ & Italian Cognitive Fusion Questionnaire \\
\hline AAQ-II & Acceptance and Action Questionnaire \\
\hline ICC & Interclass correlation coefficient \\
\hline ANOVA & Analysis of variance
\end{tabular}

\section{Declarations}

\section{Trial status}

Protocol version: Protocol Version 1.0 Date: 22/07/2020

Data collecting began in September 2020 and will be completed in Mach 2021.

\section{Ethics approval and consent to participate}

The Medical Ethic Committee of Istituto Auxologico Italiano approved the study. 
The study personnel and the Ethical Committee will ensure that the study is conducted within appropriate professional ethical guidelines, ensuring that Good Clinical Practice guidelines are observed. All participants provide written informed consent before participating in the trial or extension study.

\section{Competing interests}

The authors declare that they have no competing interests

\section{Funding}

The trial will be self-funded by the investigators.

\section{Consent for publication}

Not applicable

\section{Availability of data and material}

The datasets generated and/or analysed during the current study will be available from the corresponding author on request. Records of all patients will be kept separately in a secure place. We plan to communicate trial results to participant with a final report provided via e-mail. Result will be available for the scientific communication and health care experts via publication on scientific journal and participation into national and international congresses.

\section{Authors' contributions}

AGU and RC conceived the study, planned the experimental design and made substantial contribution to the manuscript drafting. SC, IB, GV, VV, AC, VG, MB; CV, GC, AM, participated in the implementation of the study, including the allocation, enrollment and assignment of patients to interventions, and contributed greatly to the manuscript drafting. GP FVR, GMM, GC, EM participated in the study design and helped to draft the manuscript. EG defined the statistical analysis we are planning to use for the data collected and establish the sample size for the study. All authors read and approved the final manuscript.

\section{Confidentiality}

Completed informed and written consent will be delivered to the responsable of the study.

All personal informations about participant and records of all patients will be collected and preserved separately, in a secure place, into password-protected files kept for five years after the end of trial.

\section{Communication}

We plan to communicate trial results to participant with a final report provided via e-mail. Result will be available for the scientific communication and health care experts via publication on scientific journal and participation into national and international congresses. 
Acknowledgments

Not applicable

\section{References}

A-Tjak, J. G., Davis, M. L., Morina, N., Powers, M. B., Smits, J. A., \& Emmelkamp, P. M. (2015). A metaanalysis of the efficacy of acceptance and commitment therapy for clinically relevant mental and physical health problems. Psychother Psychosom, 84(1), 30-36. doi:10.1159/000365764

Andridge, R. R., Shoben, A. B., Muller, K. E., \& Murray, D. M. (2014). Analytic methods for individually randomized group treatment trials and group-randomized trials when subjects belong to multiple groups. Stat Med, 33(13), 2178-2190. doi:10.1002/sim.6083

Baer, R. A., Smith, G. T., Hopkins, J., Krietemeyer, J., \& Toney, L. (2006). Using self-report assessment methods to explore facets of mindfulness. In (Vol. 13). Assessment.

Baroni, D., Matera C., Nerini, A., \& Stefanile, C. (2017). Adattamento Italiano del Committed Action Questionnaire-8 (CAQ-8). In. Poster session presentation at First Italian Cogress Third Wave Cognitive Behavioral Psychotherapies, Milan

Baroni, D., McCracken, L. M., Matera, C., Nerini, A., \& Stefanile, C. (2019). Misurare i valori: proprietà psicometriche della versione italiana del Brief Values Inventory. . In (Vol. 25). Psicoterapia Cognitiva e Comportamentale.

Biglan, A., \& Hayes, S. C. (1996). Should the behavioral sciences become more pragmatic? The case for functional contextualism in research on human behavior. . In (Vol. 5, pp. 47-57). Applied and Preventive Psychology.

Boffito, S., Bonalume, L., Rivolta, L., Giovannini, C., Giromini, L., \& Amadei, G. (2009). Validazione Italiana del Five Facet Mindfulness Questionnaire (FFMQ) In Congresso Nazionale della Sezione di Psicologia Clinica e Dinamica . In.

Bond, F. W., Hayes, S. C., Baer, R. A., Carpenter, K. M., Guenole, N., Orcutt, H. K., . . Zettle, R. D. (2011). Preliminary psychometric properties of the Acceptance and Action Questionnaire-II: a revised measure of psychological inflexibility and experiential avoidance. Behav Ther, 42(4), 676-688.

doi:10.1016/j.beth.2011.03.007

Butryn, M. L., Forman, E. M., Lowe, M. R., Gorin, A. A., Zhang, F., \& Schaumberg, K. (2017). Efficacy of environmental and acceptance-based enhancements to behavioral weight loss treatment: The ENACT trial. Obesity (Silver Spring), 25(5), 866-872. doi:10.1002/oby.21813

Butryn, M. L., Webb, V., \& Wadden, T. A. (2011). Behavioral treatment of obesity. Psychiatr Clin North Am, 34(4), 841-859. doi:10.1016/j.psc.2011.08.006

Page 14/19 
Castelnuovo, G., Manzoni, G. M., Villa, V., Cesa, G. L., Pietrabissa, G., \& Molinari, E. (2011). The STRATOB study: design of a randomized controlled clinical trial of Cognitive Behavioral Therapy and Brief Strategic Therapy with telecare in patients with obesity and binge-eating disorder referred to residential nutritional rehabilitation. Trials, 12, 114. doi:10.1186/1745-6215-12-114

Cattivelli, R., Manzoni , G. M., Musetti , A., Vailati Riboni, F., Bastoni, I., Guerrini Usubini, A., Pietrabissa, G., Granese, V., Maffini , N., Villa,V., Caretti, A., Varallo, G., Consoli, S., Spatola, C.M., Giusti, E.,Castelnuovo, Molinari, E. (2020). ACTonFOOD protocol. Acceptance and Awareness Based Behavioral intervention compared to a Standard Behavioral Cognitive in the treatment of obesity: an Individually Randomized Group Treatment Trial (Under review). In. Unpublished article.

Cattivelli, R., Pietrabissa, G., Ceccarini, M., Spatola, C. A., Villa, V., Caretti, A., . . Castelnuovo, G. (2015). ACTonFOOD: opportunities of ACT to address food addiction. Front Psychol, 6, 396. doi:10.3389/fpsyg.2015.00396

Chiappelli, M., Coco, G. L., Gullo, S., Bensi, L., \& Prestano, C. ( (2008)). The Outcome Questionnaire 45.2. Italian validation of an instrument for the assessment of phychological treatments. . In (Vol. 17, pp. 152161.). Epidemiology and Psychiatric Sciences.

Chorpita, B. F., Daleiden, E. L., \& Weisz, J. R. (2005). Identifying and selecting the common elements of evidence based interventions: a distillation and matching model. Ment Health Serv Res, 7(1), 5-20. doi:10.1007/s11020-005-1962-6

Chorpita, B. F., Daleiden, E. L., \& Weisz, J. R. (2005a). Modularity in the design and application of therapeutic interventions. In (Vol. 11, pp. 141-156). A pplied and Preventive Psychology.

Daltry, R. M. (2015). A case study: An ACT stress management group in a university counseling center. . In (Vol. 29, pp. 36-43.). Journal of College Student Psychotherapy.

Donner, A., Birkett, N., \& Buck, C. ( 1981). Randomization by cluster: sample size requirements and analysis. . In (Vol. 114, pp. 906-914.). American journal of epidemiology.

Dupuy, H. J. (1984). The psychological general well-being (PGWB) index. . In (pp. 1 70-183.). Assessment of quality of life in clinical trials of cardiovascular therapies.

Fairburn, C. G., \& Rothwell, E. R. (2015). Apps and eating disorders: A systematic clinical appraisal. Int J Eat Disord, 48(7), 1038-1046. doi:10.1002/eat.22398

Faul, F., Erdfelder, E., Lang, A. G., \& Buchner, A. (2007). G* Power 3: A flexible statistical power analysis program for the social, behavioral, and biomedical sciences. . In (Vol. 39 (2), pp. 175-191.). Behavior research methods.

Forman, Butryn, M. L., Manasse, S. M., Crosby, R. D., Goldstein, S. P., Wyckoff, E. P., \& Thomas, J. G. (2016). Acceptance-based versus standard behavioral treatment for obesity: Results from the mind your 
health randomized controlled trial. Obesity (Silver Spring), 24(10), 2050-2056. doi:10.1002/oby.21601

Gillanders, D. T., Bolderston, H., Bond, F. W., Dempster, M., Flaxman, P. E., Campbell, L., \& Masley, S. (2014). The development and initial validation of the cognitive fusion questionnaire. In (Vol. 45, pp. 83-101). Behavior therapy.

Giusti, E. M., Spatola, C. A., Brunani, A., Kumbhare, D., Oral, A., llieva, E., . . Capodaglio, P. (2020). ISPRM/ESPRM Guidelines on Physical and Rehabilitation Medicine (PRM) professional practice for adults with obesity and related comorbidities. Eur J Phys Rehabil Med. doi:10.23736/S19739087.20.06232-2

Glover, N. G., Sylvers, P. D., Shearer, E. M., Kane, M. C., Clasen, P. C., Epler, A. J., . . Jakupcak, M. (2016). The efficacy of Focused Acceptance and Commitment Therapy in VA primary care. Psychol Serv, 13(2), 156-161. doi:10.1037/ser0000062

Grossi, E., Groth, N., Mosconi, P., Cerutti, R., Pace, F., Compare, A., \& Apolone, G. (2006). Development and validation of the short version of the Psychological General Well-Being Index (PGWB-S). Health Qual Life Outcomes, 4, 88. doi:10.1186/1477-7525-4-88

Hayes, S. C., Strosahl, K. D., \& Wilson, K. G. (2011). Acceptance and commitment therapy: The process and practice of mindful change. In: Guilford Press.

Hayes, S. C., Gifford, E. V., \& Ruckstuhl Jr, L. E. (1996). Relational frame theory and executive function: A behavioral approach. In.

Hayes, S. C., \& Hofmann, S. G. (2017). The third wave of cognitive behavioral therapy and the rise of process-based care. World Psychiatry, 16(3), 245-246. doi:10.1002/wps.20442

Hayes, S. C., Levin, M. E., Plumb-Vilardaga, J., Villatte, J. L., \& Pistorello, J. (2013). Acceptance and commitment therapy and contextual behavioral science: examining the progress of a distinctive model of behavioral and cognitive therapy. Behav Ther, 44(2), 180-198. doi:10.1016/j.beth.2009.08.002

Hayes, S. C., Luoma, J. B., Bond, F. W., Masuda, A., \& Lillis, J. (2006). Acceptance and commitment therapy: model, processes and outcomes. Behav Res Ther, 44(1), 1-25. doi:10.1016/j.brat.2005.06.006

Hayes, S. C., \& Wilson, K. G. (1994). Acceptance and commitment therapy: Altering the verbal support for experiential avoidance. Behav Anal, 17(2), 289-303.

Hofmann, S. G., Hayes, S. C. (2019). The future of intervention science: Process-based therapy. In (Vol. 7, pp. 37-50.). Clinical Psychological Science.

Lambert, M. J., Gregersen, A. T., \& Burlingame, G. M. (2004). The Outcome Questionnaire-45. In. 
Levin, E, M., Herbert, J. D., \& Forman, E. M. (2017). Acceptance and commitment therapy: a critical review to guide clinical decision making. In. Treatments for

psychological problems and syndromes.

Levin, M. E., Krafft, J., \& Twohig, M. P. (2020). Examining processes of change in an online acceptance and commitment therapy dismantling trial with distressed college students. . In. Journal of Contextual Behavioral Science.

Linardon, J., de la Piedad Garcia, X., \& Brennan, L. (2017). Predictors, Moderators, and Mediators of Treatment Outcome Following Manualised Cognitive-Behavioural Therapy for Eating Disorders: A Systematic Review. Eur Eat Disord Rev, 25(1), 3-12. doi:10.1002/erv.2492

McCracken, L. M. (2013). Committed action: an application of the psychological flexibility model to activity patterns in chronic pain. In (Vol. 14, pp. 828-835). Pain.

McCracken, L. M., \& Yang, S.-Y. (2006). The role of values in a contextual cognitive-behavioral approach to chronic pain. In (Vol. 123, pp. 137-145.). Pain.

Oppo, A., Prevedini, A. B., Dell'Orco, F., Dordoni, P., Presti, G., Gillanders, D. T., \& Moderato, P. (2019). Fusione e Defusione. Adattamento e proprietà psicometriche della versione italiana del Cognitive Fusion Questionnaire (I-CFQ). . In (Vol. 25). Psicoterapia Cognitiva e Comportamentale.

Palavras, M. A., Hay, P., Mannan, H., da Luz, F. Q., Sainsbury, A., Touyz, S., \& Claudino, A. M. (2020). Integrated weight loss and cognitive behavioural therapy (CBT) for the treatment of recurrent binge eating and high body mass index: a randomized controlled trial. Eat Weight Disord. doi:10.1007/s40519-02000846-2

Pals, S. L., Murray, D. M., Alfano, C. M., Shadish, W. R., Hannan, P. J., \& Baker, W. L. (2008). Individually randomized group treatment trials: a critical appraisal of frequently used design and analytic approaches. Am J Public Health, 98(8), 1418-1424. doi:10.2105/AJPH.2007.127027

Pennato, T., Berrocal, C., Bernini, O., \& Rivas, T. (2013). Italian Version of the Acceptance and Action Questionnaire-II (AAQ-II): Dimensionality, Reliability, Convergent and Criterion Validity. Journal of Psychopathology and Behavioral Assessment(35), 552. doi:10.1007/s10862-013-9355-4

Riva, G., Bacchetta, M., Cesa, G., Conti, S., Castelnuovo, G., Mantovani, F., \& Molinari, E. (2006). Is severe obesity a form of addiction? Rationale, clinical approach, and controlled clinical trial. Cyberpsychol Behav, 9(4), 457-479. doi:10.1089/cpb.2006.9.457

Rotheram-Borus, M. J., Swendeman, D., Chorpita, B. F. (2012). Disruptive innovations for designing and diffusing evidence-based interventions. . In (Vol. 67). American Psychologist. 
Smout, M. F., Hayes, L., Atkins, P. W., Klausen, J., \& Duguid, J. E. (2012). The empirically supported status of acceptance and commitment therapy: An update. In (Vol. 16, pp. 97-109.): Clinical Psychologist, .

Strosahl, K, Robinson, P., \& Gustavsson, T. (2012). Brief interventions for radical change: Principles \& practice of focused acceptance \& commitment therapy. . In. Oakland, CA: New Harbinger Publications.

Villatte, J. L., Vilardaga, R., Villatte, M., Plumb Vilardaga, J. C., Atkins, D. C., \& Hayes, S. C. (2016). Acceptance and Commitment Therapy modules: Differential impact on treatment processes and outcomes. Behav Res Ther, 77, 52-61. doi:10.1016/j.brat.2015.12.001

Wing, R. R., \& Hill, J. O. (2001). Successful weight loss maintenance. In (Vol. 21, pp. 323-341.). Annual review of nutrition.

Wing, R. R., Tate, D. F., Espeland, M. A., Lewis, C. E., LaRose, J. G., Gorin, A. A., . . Group, S. o. N. A. t. W. G. P. S. R. (2016). Innovative Self-Regulation Strategies to Reduce Weight Gain in Young Adults: The Study of Novel Approaches to Weight Gain Prevention (SNAP) Randomized Clinical Trial. JAMA Intern Med, 176(6), 755-762. doi:10.1001/jamainternmed.2016.1236

Yumuk, V., Tsigos, C., Fried, M., Schindler, K., Busetto, L., Micic, D., ... Obesity, O. M. T. F. o. t. E. A. f. t. S. o. (2015). European Guidelines for Obesity Management in Adults. Obes Facts, 8(6), 402-424. doi:10.1159/000442721

\section{Figures}




\begin{tabular}{|c|c|c|c|c|c|c|}
\hline \multirow[b]{2}{*}{ TIMEPOINT** } & \multirow{2}{*}{$\begin{array}{c}\text { Enrolment } \\
-t_{l}\end{array}$} & \multirow{2}{*}{$\begin{array}{c}\text { Allocation } \\
0\end{array}$} & \multicolumn{2}{|c|}{ Intervention } & \multicolumn{2}{|c|}{ Follow-up } \\
\hline & & & 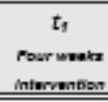 & $\begin{array}{c}1 \text { mants } \\
\text { mantaring } \\
\end{array}$ & $t_{2}^{t_{2}}$ & $\begin{array}{c}T_{3} \\
12 \text { monthx }\end{array}$ \\
\hline \multicolumn{7}{|l|}{ ENROLMENT: } \\
\hline \multirow{2}{*}{$\begin{array}{l}\text { Eligibility screen } \\
\text { Informed consent }\end{array}$} & $x$ & & & & & \\
\hline & $\mathrm{X}$ & & & & & \\
\hline Allocation & & $\mathrm{X}$ & & & & \\
\hline \multicolumn{7}{|l|}{ INTERVENTIONS: } \\
\hline \multicolumn{7}{|l|}{ [Module Engagement] } \\
\hline \multicolumn{7}{|l|}{ [Module Awareness] } \\
\hline \multicolumn{7}{|l|}{ [Module Acceptance] } \\
\hline \multicolumn{7}{|l|}{ ASSESSMENTS: } \\
\hline 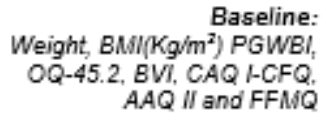 & & $\mathrm{x}$ & & & & \\
\hline 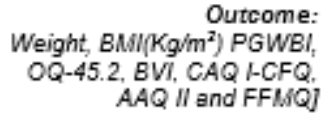 & & & $\mathrm{x}$ & & $\mathrm{X}$ & $\mathrm{x}$ \\
\hline $\begin{array}{r}\text { Weight loss maintenance: } \\
\text { total amount of moderate- } \\
\text { to-vigorous physical } \\
\text { activity in minutes; } \\
\text { weekly mean of daily total } \\
\text { amount of calories; } \\
\text { weekly mean of total daily }\end{array}$ & & & & $x$ & & \\
\hline
\end{tabular}

•Recommended content can be displayed using various schematic formats. See SPIRIT 2013

Explanation and Elaboration for examples from protocols.

*List specific timepoints in this row.

\section{Figure 1}

Example template of recommended content for the schedule of enrolment, interventions, and assessments.*

\section{Supplementary Files}

This is a list of supplementary files associated with this preprint. Click to download.

- SPIRITChecklistdownload8Jan13.doc 